\section{Requirement for math5 in the development of retinal ganglion cells}

\author{
Steven W. Wang, ${ }^{1,3}$ Byong Su Kim, ${ }^{1,3}$ Kan Ding, ${ }^{2,3}$ \\ Huan Wang, ${ }^{2}$ Dantong Sun, ${ }^{1}$ Randy L. Johnson, ${ }^{1}$ \\ William H. Klein, ${ }^{1}$ and Lin Gan ${ }^{1,2,4}$
}

${ }^{1}$ Department of Biochemistry and Molecular Biology, The University of Texas M. D. Anderson Cancer Center, Houston, Texas 77030, USA; ${ }^{2}$ Center for Aging and Developmental Biology, Box 645, University of Rochester, Rochester, New York 14642, USA

math 5 is a murine orthologue of atonal, a bHLH proneural gene essential for the formation of photoreceptors and chordotonal organs in Drosophila. The expression of math 5 coincides with the onset of retinal ganglion cell (RGC) differentiation. Targeted deletion of math5 blocks the initial differentiation of $80 \%$ of RGCs and results in an increase in differentiated amacrine cells. Furthermore, the absence of math 5 abolishes the retinal expression of $b r n-3 b$ and the formation of virtually all brn- $3 b$ expressing RGCs. These results imply that math5 is a proneural gene essential for RGC differentiation and that math 5 acts upstream to activate brn-3b-dependent differentiation processes in RGCs.

Received September 27, 2000; revised version accepted November 20, 2000.

The mammalian retina is the peripheral portion of the visual system containing six major neuronal cell types and one glial cell type organized in a laminar structure. The visual information process in the retina follows a general pathway from photoreceptors to bipolar cells to retinal ganglion cells (RGCs). Horizontal cells and amacrine cells act to mediate lateral interactions among photoreceptors, bipolar cells, and RGCs. The latter serve as the sole output neurons in the retina to send the visual information down the optic nerve to the rest of brain. Both birthdating experiments using ${ }^{3} \mathrm{H}$-thymidine labeling and cell lineage analysis using retroviral and tracermediated approaches demonstrate that vertebrate retinal neurons are generated from common progenitors through sequential differentiation and ordered migration to form the laminar retinal structure (Cepko et al. 1996). Current models for retinal neuron differentiation suggest that the formation of a specific retinal neuron is deter-

\footnotetext{
[Key Words: bHLH; math5; POU-domain; brn-3b; retinal development] ${ }^{3}$ These authors contributed equally to this article.

${ }^{4}$ Corresponding author.

E-MAIL lin_gan@urmc.rochester.edu; FAX (716) 756-7665.

Article and publication are at www.genesdev.org/cgi/doi/10.1101/ $\operatorname{gad} .855301$.
}

mined by the intrinsic properties of the retinal progenitor and the extrinsic cues from the retinal environment (Cepko et al. 1996). Among the likely intrinsic factors, the basic helix-loop-helix (bHLH) class of proneural transcription factors appears to play an essential role in regulating the differentiation of retinal neurons (Cepko 1999|. In Drosophila, expression of proneural genes of the achaete-scute complex (AS-C) and atonal (ato) in proneural clusters endow cells with neural competence (Jan and Jan 1993). Loss-of-function mutation of genes in AS-C causes the cells in the would-be proneural clusters to adopt epidermal fates rather than neuronal precursor fates. Conversely, gain-of-function mutations in the proneural genes leads to the ectopic formation of sensory neurons (Campuzano and Modolell 1992). The expression of ato is found in the optic furrow of the eye-antennal disc in addition to the ectodermal proneural clusters and sensory organ precursors, which give rise to the chordotonal organs. Deletion of ato causes the absence of the chordotonal organ and the lack of photoreceptors (Jarman et al. 1995), and the reduced expression of ato results in defects in axonal pathfinding of photoreceptors (White and Jarman 2000), suggesting that ato plays dual roles in determining neuronal potential and regulating specific neuronal differentiation events. Murine orthologs of many Drosophila proneural genes, including NeuroD, mash1, math4a/Ngn2, math5, and math1, are expressed in the developing retina and are thought to function as positive regulators of neuronal differentiation programs (Brown et al. 1998; Cepko 1999). Mutagenesis studies have shown that mash1 regulates the formation of late-born retinal neurons, particularly the bipolar cells, and NeuroD acts to control the differentiation of amacrine and bipolar cells (Tomita et al. 1996; Morrow et al. 1999). While the differentiation of RGCs, the first-born retinal neurons, begins at embryonic day 11.5 (E11.5; Young 1985a), the retinal expression of NeuroD, math1, and math4a/Ngn2 genes initiate at E13.5, and the retinal expression of mash1 is not detected until E14.5 (Brown et al. 1998), indicating that none of the above bHLH genes is responsible for the initial differentiation of RGCs.

math5, an ortholog of ato, is transiently expressed in developing mouse retinas starting at E11, and its expression coincides with the differentiation of RGCs (Brown et al. 1998). Thus, math5 is a good candidate for a proneural gene required for RGC formation. To investigate the role of math5 in mouse retinal development and, particularly, in the development of RGCs, we used homologous recombination in murine embryonic stem (ES) cells to delete math5. We show here that targeted deletion of math 5 results in the loss of $>80 \%$ of RGCs and of virtually all brn-3b-expressing RGCs and leads to an increase in differentiated amacrine cells. Expression properties of a reporter $1 a c Z$ gene in math5-1acZ knock-in mice demonstrate that deletion of math5 does not prevent the formation of RGC progenitors. However, math5 deficiency leads to the loss of retinal expression of the 
early RGC differentiation markers, brn-3b and $p 75$, implying that the null mutation in math5 blocks the initial differentiation of RGCs. The results imply that math5 is essential for the initial differentiation of RGCs and that the loss of math5 is likely to cause a cell-fate conversion of retinal progenitors from RGCs to amacrine cells. The effect of the math5 mutation on the onset of brn-3b expression suggests that math5 acts upstream to activate brn-3b-dependent differentiation processes in RGCs.

\section{Results and Discussion}

Targeted deletions of math5 and retinal expression of a reporter lacZ gene

To investigate the role of math5 in vivo, we generated a targeted deletion of math5 by homologous recombination in ES cells. The mutant math5 allele was created by replacing the entire math5 open reading frame (ORF) with either a lacZ or GFP reporter gene (Fig. 1). Mice heterozygous for the math5-null allele appeared normal with no apparent retinal defects. Both heterozygous and homozygous math5-mutant mice were viable and fertile and had no discernible physical defects.

Previous in situ hybridization studies have shown that the retinal expression of math5 was initiated at E11, before the onset of RGC differentiation. Its retinal expression was often observed in cells adjacent to proliferating regions (Brown et al. 1998). To determine whether the expression pattern of the knock-in lacZ gene faithfully reflected that of endogenous math5 and to evaluate the possible effects of the math5 mutation on retinal development, we compared math5-lacZ expression in the retinas of heterozygous and homologous mutant embryos. math5-lacZ expression in retinas of heterozygous embryos was first detected at E11 and peaked at E13.3, with

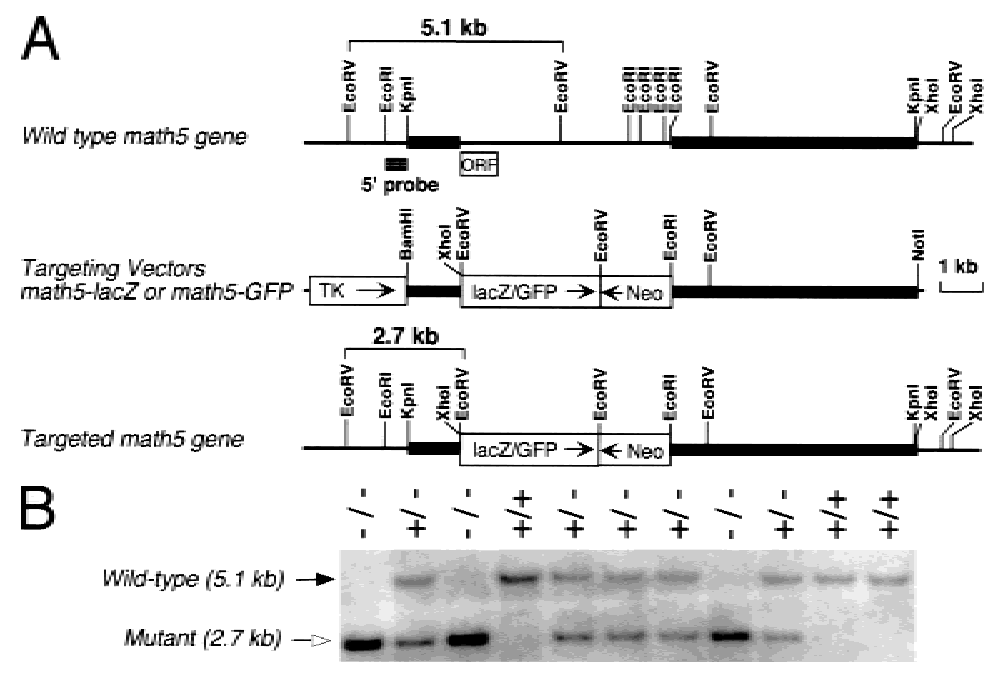

Figure 1. Targeted deletion and expression of math5. (A) math5 genomic structure, restriction enzyme map, and targeting strategy. The DNA fragment containing the math5 ORF region was replaced with GFP or lacZ in the targeting vector. Neo, PGK-neo cassette; TK, MC1-TK cassette. $(B)$ Southern genotyping of a typical litter from a heterozygous cross. The $5^{\prime}$ probe detected 5.1-kb wild-type and 2.7-kb mutant EcoRV fragments.
math5-lacZ-expressing cells distributed uniformly from the ventricular (proliferating) zone to the vitreous side (retinal ganglion layer) of the retina (Fig. 2A,C,E). After E13.5, lacZ expression was significantly reduced over the entire retina. At E15.5, robust expression was limited to the peripheral regions of the retina where RGCs were actively being generated, but much weaker expression was observed in more medial regions where RGCs were postmigratory (Fig. 2G). The lacZ expression in the ganglion cell layer (GCL) was likely to represent residual lac $Z$ activity because endogenous math5 expression is not found in differentiated RGCs (Brown et al. 1998). The overall expression pattern of lac $Z$ in heterozygous embryonic retinas closely resembled that of endogenous math5 (Brown et al. 1998) and indicated that the math5 regulatory sequences in the math5-lac Z allele were sufficient to confer correct spatiotemporal expression. No notable differences in lac $Z$ expression were seen when homozygous math5-1acZ/math5-GFP retinas from E11, E12.5, and E13.5 embryos were compared with similarly staged heterozygous math5-1acZ/+ retinas (Fig. 2A-F), suggesting that a comparable number of math5-expressing cells were generated in heterozygous and homozygous math5-mutant retinas and that the expression of math5 was not controlled by autoregulatory mechanisms as is the case for ato and math1 (Jarman et al. 1995; Helms et al. 2000). However, in math5-lacZ/ math5-GFP retinas at E15.5, we found a complete absence of the residual lacZ expression in GCL, which contrasted with the readily observable albeit weak expression in math5-1acZ/+ retinas at the same stage (Fig. $2 \mathrm{G}, \mathrm{H})$. These results indicated that very few differentiated RGCs were forming in math5-deficient retinas.

\section{Retinal ganglion cell defects of math5-null mice}

To assess the potential loss of RGCs in math5null retinas, we analyzed retinal sections prepared from postnatal mice at $3 \mathrm{wk}$ of age, a time when all retinal neurons are fully developed (Young 1985b). The overall laminar structure of the homozygous math5-deficient retinas resembled those of wild-type and heterozygous retinas, and no noticeable changes in the number of photoreceptor cells in the outer nuclear layer (ONL) were observed. However, the mutant retinas were $\sim 15 \%-20 \%$ thinner (Fig. $3 \mathrm{~A}, \mathrm{~B}$ ). This difference was mostly caused by the loss of $>40 \%$ of the cells in GCL and the absence of a defined nerve fiber layer (NFL), which consists entirely of bundles of RGC axons (Fig. 3A,B). In mice, RGCs and displaced amacrine cells each constitute about one-half of the cells in GCL (Perry 1981; Barnstable and Drager 1984). Therefore, the $40 \%$ or more loss of cells in GCL could account for a $>80 \%$ loss of RGCs. Quantification of the number of axon bundles in retinas immunostained with SMI-32, a mouse monoclonal antibody that reacts with neurofilament $\mathrm{H}$ and labels predominantly the axons of large ganglion cells 


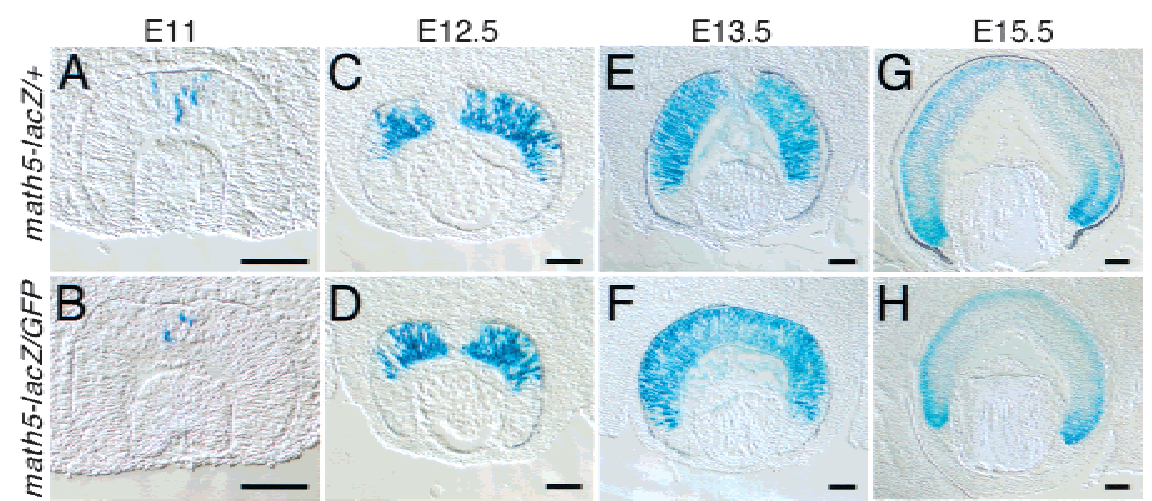

Figure 2. Expression of the math5-1acZ fusion gene in developing retinas. The math5 heterozygous (math5-lacZ/+) and homozygous (math5-lacZ/GFP) retinas were collected for whole-mount staining of $l a c Z$ activities. After staining, the retinas were cryosectioned and photographed using DIC-Nomarski optics. The math5-lacZ expression was first detected in the central region of developing retina at E11.5 $(A, B)$. At E12.5 $(C, D)$ and E13.5 $(E, F)$, math5-lacZ expression rapidly expanded throughout the retinas. After peaking at E13.5, high-level expression was limited to the peripheral regions of the retina $(G, H)$, where RGCs are being generated. Scale bar, $100 \mu \mathrm{m}$.

(Nixon et al. 1989), showed an $\sim 80 \%$ reduction of SMI32-positive processes in math5-null retinas $(118 \pm 12$ axon bundles) relative to wild-type controls $(583 \pm 24.8$ axon bundles; Fig. 3C,D). The remaining $20 \%$ of the SMI-32-positive processes appeared to form axon bundles, projected toward the optic disk region, and formed a very thin optic nerve and optic chiasm (Fig. 3D; data not shown). However, many of these axon bundles were abnormally curved, suggesting a defect in axon pathfinding during development.

In previous studies, we showed that the Class IV POU domain-containing brn-3b gene was required for the terminal differentiation of RGCs and that null mutations in brn-3b resulted in axon growth defects and programmed cell death in $\sim 70 \%$ of newly formed RGCs (Gan et al. 1996, 1999). The loss of RGCs observed in the math5deficient mice resembled that of brn-3b-null mice. To test whether math 5 and brn-3b were involved in the differentiation of the same population of RGCs, we generated mice with compound mutations in math5 (math5-GFP) and brn-3b (brn-3b-lacZ). Nuclear expression of lacZ in brn-3b-lac $Z$ mice serves to effectively mark brn-3b-positive RGCs (Gan et al. 1999). Compared with normal retinas (brn-3b-lacZ/+, math5+/+), math5-null retinas (brn-3blacZ/+, math5-GFP/math5-GFP) showed a loss of $\sim 97 \%$ of brn-3b-lacZ-expressing cells $\left(1678 \pm 26\right.$ cells $/ \mathrm{mm}^{2}$ express brn-3b-lac $Z$ in normal retinas, as compared with $47 \pm 2.6$ cells $/ \mathrm{mm}^{2}$ in math5-null retinas; Fig. 3E,F), thus demonstrating that math 5 was required for the differentiation of most brn-3b-expressing RGCs. The remaining RGCs in math5-deficient retina appeared to be expressing brn- $3 b$ but were generated by a math5-independent pathway.

\section{Absence of early RGC differentiation in math5-null retinas}

The absence of $80 \%$ of RGCs in mature math5-deficient retinas could be the result of an insufficient number of
RGC progenitors or a failure of RGC differentiation or survival. Because a similar number of math5-1acZ-expressing cells were detected in heterozygous and homologous math5mutant retinas between E11 and E15.5 (Fig. 2), it was unlikely that there were an insufficient number of progenitor cells. TUNEL analysis also revealed no overt difference in apoptosis in retinas at E12.5-E19 in math5-deficient and wild-type embryos (data not shown). A reasonable hypothesis was that math5-deficient retinas were unable to form RGCs. To test whether math5-null retinal progenitor cells failed to differentiate into RGCs, we examined the presence of newly differentiated RGCs by detecting the expression of the early RGC markers, brn- $3 b$ and $p 75$, the latter of which encodes a receptor for nerve growth factor (NGF) and neurotrophins (Frade and Barde 1999; Gan et al. 1999). Section in situ hybridization with a brn-3b antisense probe and expression of the brn-3b-lacZ allele at E13.5 showed that brn-3b was highly expressed in wild-type retinas (Fig. 4C,E) but was virtually absent in homozygous math5-null retinas (Fig. $4 \mathrm{D}, \mathrm{F})$. Similarly, immunostaining of E13.5 retinas with anti-p75 showed that the expression of $p 75$ in math5deficient retina was severely reduced (Fig. 4G,H). The absence of most RGCs in mature math5-deficient retinas, and the lack of brn-3b and $p 75$ expression in math5deficient embryonic retinas, argued strongly that in math5-null retinas, retinal progenitor cells were unable to differentiate into RGCs. Because brn-3b expression was essentially absent in math5-null retinas, the results also demonstrated that math5 was genetically upstream of brn-3b.

\section{Effects of math5 mutation on other retinal neurons}

In Drosophila, ato is required for the specification of the founder photoreceptors - the R8 cells, which in turn recruit the other photoreceptors (Jarman et al. 1995). Current models of retinal neuron differentiation suggest that the cell differentiation of RGCs is negatively regulated through a feedback mechanism by the presence of differentiated RGCs (Cepko et al. 1996; Belliveau and Cepko 1999; Belliveau et al. 2000). However, it remains unclear how the loss of RGCs could influence the formation of other retinal neurons. In addition, the lack of appropriate synaptic connections in the absence of most RGCs is likely to have pronounced effects on neurons in the inner nuclear layer (INL). To determine whether the math5 mutation affected the development of retinal neurons other than RGCs, we monitored the expression of Protein Kinase C- $\alpha$ (PKC- $\alpha$ ) as a bipolar cell marker (Wassle and Boycott 1991) and syntaxin and choline acetyltransferase (ChAT) as amacrine cell markers (Barnstable et al. 1985; Jeon et al. 1998) in mature retina. 

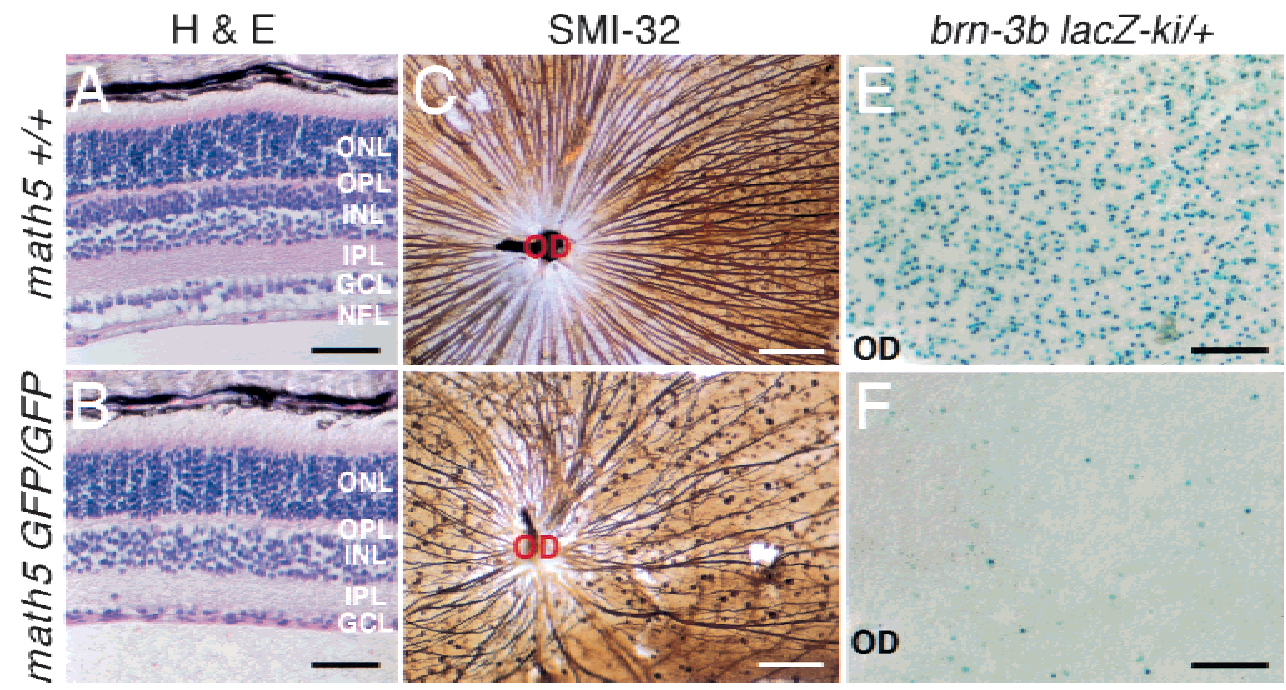

Figure 3. Reduction in the number of retinal ganglion cells in math5-mutant retinas. Retinas from 3-wk-old math5 wild-type $($ math5+/+) and mutant (math5 GFP/GFP) mice were analyzed by haematoxylin and eosin (H\&E) staining $(A, B)$, whole-mount immunostaining with SMI-32 $(C, D)$, and X-Gal staining for brn-3b-lacZ expression $(E, F)$. Retinal neurons are organized in a layered structure with photoreceptors (cones and rods) located in the outer nuclear layer (ONL), interneurons (bipolar, horizontal, and amacrine cells) in the inner nuclear layer (INL), and retinal ganglion cells (RGCs) and displaced amacrine cells in the ganglion cell layer (GCL). The synaptic contacts among retinal neurons are restricted to two defined layers. The outer plexiform layer (OPL) contains the synaptic contacts among photoreceptor, bipolar, and horizontal cells, and the inner plexiform layer (IPL) consists of the synaptic connections among bipolar, amacrine, and ganglion cells. Ganglion nerve fiber layer (NFL) consists of axons of RGCs. In math5mutant retinas, the thickness of the ONL remains unchanged. However, the number of cells in the GCL dropped significantly, and the thickness of NFL was greatly reduced $(B)$. SMI-32 and lacZ expression revealed the loss of $>80 \%$ of total RGCs and most of the brn-3b-expressing RGCs in mutant retinas $(C-F)$. OD, optic disc. Scale bar, $50 \mu \mathrm{m}$ in $A$ and $B, 100 \mu \mathrm{m}$ in $C$ and $D$, and $200 \mu \mathrm{m}$ in $E$ and $F$.

PKC- $\alpha$ is expressed in cell bodies and processes of bipolar cells that mainly connect to rods (Wassle and Boycott 1991). In wild-type retinas, PKC- $\alpha$ labeled cells in
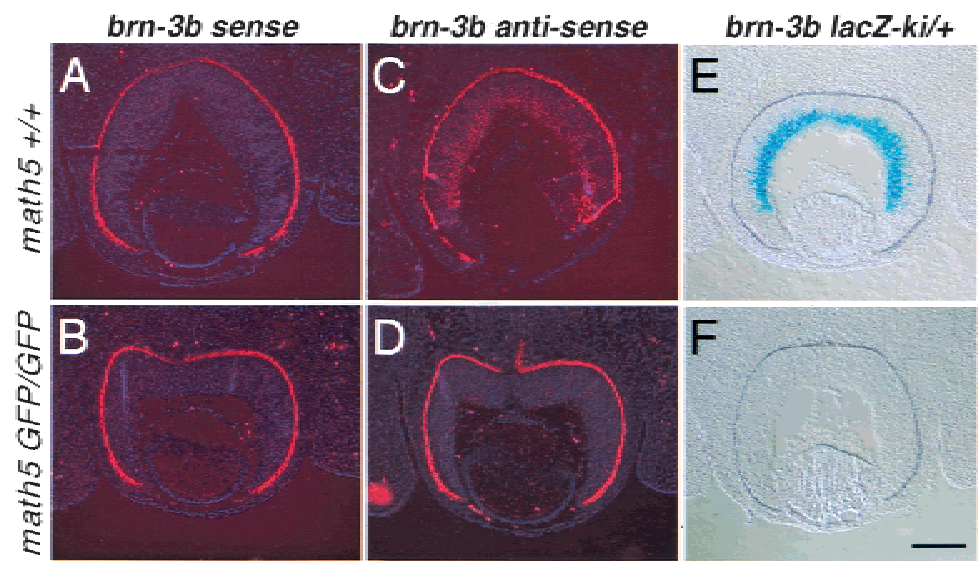

Figure 4. Absence of newborn RGCs in math5-null retinas at E13.5. Retinal sections from wild-type $(A, C)$ and math5-null $(B, D)$ mice were probed with brn-3b sense $(A, B)$ and antisense $(C, D)$ probes in sectioned in situ hybridization. brn-3b was expressed at high levels in the RGC layer in wild-type retina $(C)$, but its expression was severely reduced in math5-null retina $(D)$. brn-3b sense probe served as an in situ hybridization control. Similarly, the expression of brn-3b-lac $Z$ was in heterozygous brn-3b lac $Z$ retinas in a math5 wild-type background $(E)$ but not in math5-null background $(F)$. Immunostaining with anti-p75 (green) revealed the accumulation of p75 in newly formed RGCs in wild-type retina at E13.5 $(G)$ but not in math5-deficient retina $(H)$. Nuclei in $G$ and $H$ were counterstained with propidium iodide (red). Scale bar, $200 \mu \mathrm{m}$ in $A-F$ and $50 \mu \mathrm{m}$ in $G$ and $H$.
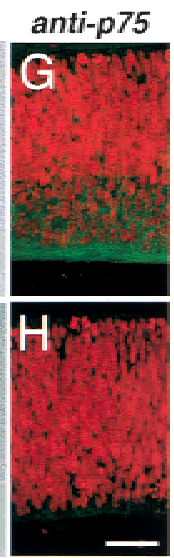

the inner nuclear layer and axons and dendrites within the outer and inner plexiform layer (Fig. 5A). In math5mutant retina, the labeling pattern and the number of PKC- $\alpha$-expressing cells were similar to those in the wild-type retinas, indicating that math5-null mutation had no apparent effects on the bipolar cells expressing PKC- $\alpha$.

In contrast to bipolar cells, amacrine cells were noticeably affected by the loss of math5. Expression of both syntaxin and ChAT were strongly upregulated when compared to wild-type controls (Fig. 5C-F). Quantitation of the number of ChAT-positive cells demonstrated a 7.5-fold increase in math5-null retinas (93 \pm 12 ChAT-expressing cells/ $0.04 \mathrm{~mm}^{2}$ ) relative to wild-type controls $\quad(12.3 \pm 3.3 \quad$ ChAT-expressing cells $/ 0.04 \mathrm{~mm}^{2}$ ). Moreover, most of the syntaxin- and ChAT-positive cells were located in GCL rather than the inner nuclear layer. In vertebrates, starburst amacrine cells are the only cholinergic retinal cells, and the cholinergic amacrine cells in GCL are the ON-starburst amacrine cells. Our data suggested that the loss 


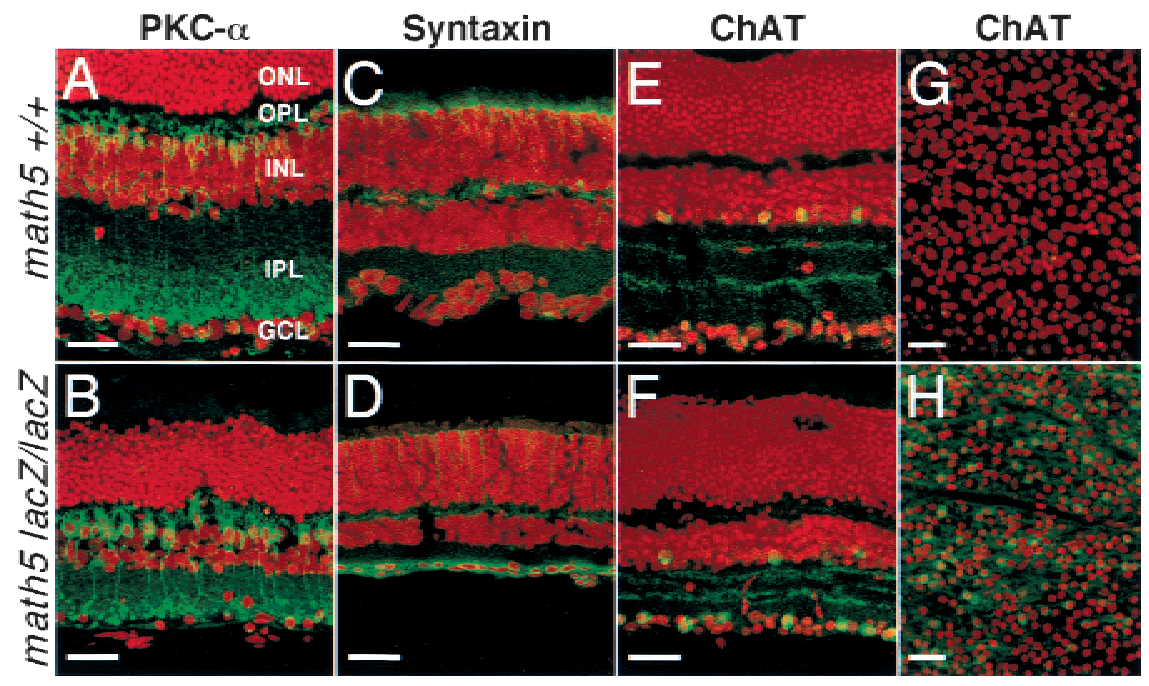

Figure 5. Effect of the math5-null mutation on bipolar and amacrine cells in adult retinas. Retinas were immunolabeled with antibodies (green) and counter-stained for nuclei with propidium iodide (red). No significant changes were detected in math5-null retina in the number of bipolar cells as shown by anti-PKC- $\alpha$ expression $(A, B)$. Antisyntaxin $(C, D)$ and anti-ChAT $(E, F$, and in whole-mount, $G, H)$ labeling demonstrated an increased number of amacrine cells in math5-null retinas, in particular a 7.5-fold increase in the number of cholinergic amacrine cells labeled by anti-ChAT. Scale bar, $30 \mu \mathrm{m}$.

of math5 resulted in an increase of amacrine cells, in particular the ON-starburst amacrine cells, and their displacement to GCL. Most likely, the displacement of amacrine cells was caused by the loss of retinal ganglion cells. Based on cell birthdating experiments, amacrine cells are among the early-born neurons in the mouse retina and are generated at approximately the same time as RGCs (Cepko et al. 1996). Thus, it is possible that in math5-null retinas, progenitor cells unable to form RGCs switch their fate to the amacrine cell differentiation pathway. It is also possible that math5 is expressed in amacrine cell lineages during normal retinal development and negatively controls the differentiation of amacrine cells. However, if math5 acts like the other atonalclass bHLH proneural genes to promote the differentiation of specific neurons, it is most likely that math5 is expressed only in RGC lineages and promotes their differentiation rather than in amacrine cell lineages to inhibit their differentiation.

Given that the majority of RGCs did not differentiate at early stages of retinal development, the fact that the resulting retina maintained its structural integrity and contained all other neuronal cell types was unexpected. Our results indicated that the developmental relationships among the retinal cell types are not tightly regulated and that the lack of majority of RGCs does not dramatically affect the overall patterning of the retina.

Several investigations have shown that the NotchDelta pathway is important for negatively regulating the differentiation of RGCs and other neurons in the retina (Austin et al. 1995; Dorsky et al. 1995; Bao and Cepko 1997). The Notch-Delta pathway likely acts to negatively regulate the expression of proneural genes like math5 in the mouse retina, as is the case for ato in the Drosophila eye. Because math5 encodes a bHLH transcriptional factor, it must function by regulating genes required for RGC differentiation. In Drosophila, ato functions to regulate the formation and axon pathfinding properties of R8 photoreceptors (White and Jarman 2000). However, the molecular mechanisms of its function in axon pathfinding and other neural terminal differentiation processes remain poorly understood. Our studies showed that math5- and brn-3b-null mutations affect the development of largely the same population of RGCs and that math5 appears to be genetically upstream of brn-3b. Combined with our early studies that brn-3b is involved in normal axon growth of mouse RGCs (Gan et al. 1999; Wang et al. 2000), our results indicate that although the morphological and physiological properties of R8 photoreceptors in Drosophila and RGCs in mice are highly diverged, a conserved genetic regulatory pathway may control the differentiation of both of these cell types. The down-regulation of the Notch-Delta signaling pathway leads to the up-regulation of ato/math5, which in turn activates downstream effector genes like brn-3b to promote R8/RGC differentiation and normal axonal outgrowth. It remains to be determined whether math 5 activates brn-3b directly during normal retinal development. The results presented here demonstrate that the proneural gene math5 is essential for the differentiation of RGCs and that a null mutation of math 5 results in the failure of the majority of RGCs to form.

Previous studies have shown that targeted expression of Xath5, a math5 ortholog in Xenopus, in the optic vesicle promotes the generation of RGCs, suggesting that Xath5 is sufficient to direct the differentiation of retinal progenitors into RGCs (Kanekar et al. 1997). Whether or not math5 is sufficient for RGC in the mouse can be tested by ectopic expression of math5 in retinal progenitors. In addition, while ato is required for the generation of all R8 cells, math 5 was essential for the appearance of the majority of RGCs. It is likely that in mouse, other genes, particularly other proneural $b H L H$ genes expressed in the retina, could account for the remaining RGCs.

\section{Materials and methods}

\section{Targeted deletion of math5}

Mouse math5 genomic sequences were isolated from a mouse genetic DNA library (Strain 129/SvEv) in $\lambda$ DASHII (Stratagene), using math5 cDNA (L. Gan, unpubl.) as a probe. Targeted mutation of math5 was generated by inserting the math $1.5-\mathrm{kb} 5^{\prime}$ sequences containing the entire $5^{\prime}$ untranslated region and the $5.8-\mathrm{kb} 3^{\prime}$ sequences into the BamHI/XhoI and EcoRI/NotI sites in the $5^{\prime}$ and $3^{\prime}$ multiple cloning sites 
of pKI-lacZ or pKI-GFP vectors (L. Gan, unpubl.), respectively. The constructs place the lacZ and GFP under the control of math5 regulatory sequences. Generation of mutant mice, genotyping, and X-Gal staining were done as described (Gan et al. 1996, 1999). All analysis was done on a mixed C57B6/J and 129/SvEv background. To compare the number of brn-3b-lacZ-expressing cells, three math5 wild-type and three math5 mutant retinas from two litters at 3 wk old were whole-mount stained for $1 a c Z$ activities, and the number of brn-3b-lac $Z$ positive cells were counted in an $800 \times 1200 \mu \mathrm{m}$ field adjacent to the optic disc.

\section{Sectioned in situ hybridization}

Sectioned in situ hybridization was conducted as described (Riddle et al. 1993). To generate brn-3b probes, the mouse 3.1-kb brn-3b cDNA clone was isolated from a mouse E14.5 retina cDNA library (L. Gan, unpubl.) in pBluescript SKII ++ ) (Strategene). The cDNA clone was linearized with $X h o I$ and $X b a I$ for synthesis of sense and antisense probes using T3 and T7 RNA polymerase, respectively.

\section{Immunohistochemistry and microscopy}

Immunohistochemical staining of whole-mount retinas using SMI-32 (Sternberger Monoclonals) was done as described (Gan et al. 1996). Counts of SMI-32-expressing RGCs were compared in four wild-type and three math 5 mutant retinas from mice at 3 wk old. To quantify the number of SMI-32-positive RGCs, micrographs of the whole-mount stained retinas were taken and the total numbers of axon bundles were counted at the circumference $\sim 500 \mu \mathrm{m}$ from the optic disc.

For confocal microscopy, retinal tissues from different ages were fixed with $3.2 \%$ paraformaldehyde in $\mathrm{PBS}(\mathrm{pH} 7.4)$ for $30 \mathrm{~min}$ at room temperature. Fixed samples were washed three times with PBS and cryosectioned to a thickness range from 15 to $25 \mu \mathrm{m}$. Sections were incubated 30 min in PBS containing $0.05 \%$ Triton X-100 (PBS-T) and blocked with $2 \%$ BSA in PBS-T for $1 \mathrm{~h}$. Sections were then incubated sequentially for $1 \mathrm{~h}$, each with the primary antibody and secondary antibody. Sections were washed three times with PBS-T between the antibody incubations. Primary antibodies used were mouse antirat syntaxin (Sigma, S0664), rabbit antihuman choline acetyltransferase (Chemicon, AB143), rabbit antimouse PKC- $\alpha$ (Sigma, P4334), and rabbit antihuman p75 (Promega, G323A). Secondary antibodies used were Alexa 488-conjugated antimouse or antirabbit IgG (Molecular Probes). Antibody-labeled sections were treated with $0.05 \%$ propidium iodide for $2 \mathrm{~min}$ at room temperature. Prepared sections were mounted in Fluoromount (EMS) and examined using a Zeiss 410 or a Olympus FV300 confocal microscope. Images were projected from eight optical sections with intervals of $0.5 \mu \mathrm{m}$. Projected images were pseudocolored to restore the colors as seen in an epifluorescent microscope. To quantify the number of ChAT-positive amacrine cells, three wild-type and three math5-mutant retinas from the same litter were whole-mount stained with anti-ChAT antibody, and the counts were done on micrographs in a $200 \times 200 \mu \mathrm{m}$ field.

\section{Acknowledgments}

We thank Q. Fu and M. Wu for technical asistance and Jose Luis de la Pompa for critical reading of the manuscript. This work was supported by the Silbermann Foundation and Retinal Research Foundation (L.G.) and an NEI grant RO1EY11930 and the Welch Foundation (W.H.K.).

The publication costs of this article were defrayed in part by payment of page charges. This article must therefore be hereby marked "advertisement" in accordance with 18 USC section 1734 solely to indicate this fact.

\section{References}

Austin, C.P., Feldman, D.E., Ida, Jr., J.A., and Cepko, C.L. 1995. Vertebrate retinal ganglion cells are selected from competent progenitors by the action of Notch. Development 121: 3637-3650.

Bao, Z.Z. and Cepko, C.L. 1997. The expression and function of Notch pathway genes in the developing rat eye. J. Neurosci. 17: 1425-1434.

Barnstable, C.J. and Drager, U.C. 1984. Thy-1 antigen: A ganglion cell specific marker in rodent retina. Neuroscience 11: 847-855.

Barnstable, C.J., Hofstein, R., and Akagawa, K. 1985. A marker of early amacrine cell development in rat retina. Brain Res. 352: 286-290.

Belliveau, M.J. and Cepko, C.L. 1999. Extrinsic and intrinsic factors con- trol the genesis of amacrine and cone cells in the rat retina. Development 126: 555-566.

Belliveau, M.J., Young, T.L., and Cepko, C.L. 2000. Late retinal progenitor cells show intrinsic limitations in the production of cell types and the kinetics of opsin synthesis. J. Neurosci. 20: 2247-2254.

Brown, N.L., Kanekar, S., Vetter, M.L., Tucker, P.K., Gemza, D.L., and Glaser, T. 1998. Math5 encodes a murine basic helix-loop-helix transcription factor expressed during early stages of retinal neurogenesis. Development 125: 4821-4833.

Campuzano, S. and Modolell, J. 1992. Patterning of the Drosophila nervous system: The achaete-scute gene complex. Trends Genet. 8: 202208.

Cepko, C.L. 1999. The roles of intrinsic and extrinsic cues and bHLH genes in the determination of retinal cell fates. Curr. Opin. Neurobiol. 9: 37-46.

Cepko, C.L., Austin, C.P., Yang, X., Alexiades, M., and Ezzeddine, D. 1996. Cell fate determination in the vertebrate retina. Proc. Natl. Acad. Sci. 93: 589-595.

Dorsky, R.I., Rapaport, D.H., and Harris, W.A. 1995. Xotch inhibits cell differentiation in the Xenopus retina. Neuron 14: 487-496.

Frade, J.M. and Barde, Y.A. 1999. Genetic evidence for cell death mediated by nerve growth factor and the neurotrophin receptor p75 in the developing mouse retina and spinal cord. Development 126: 683-690.

Gan, L., Xiang, M., Zhou, L., Wagner, D.S., Klein, W.H., and Nathans, J. 1996. POU domain factor Brn-3b is required for the development of a large set of retinal ganglion cells. Proc. Nat1. Acad. Sci. 93: 39203925.

Gan, L., Wang, S.W., Huang, Z., and Klein, W.H. 1999. POU domain factor Brn-3b is essential for retinal ganglion cell differentiation and survival but not for initial cell fate specification. Dev. Biol. 210: 469-480.

Helms, A.W., Abney, A.L., Ben-Arie, N., Zoghbi, H.Y., and Johnson, J.E. 2000. Autoregulation and multiple enhancers control Math1 expression in the developing nervous system. Development 127: $1185-1196$.

Jan, Y.N. and Jan, L.Y. 1993. HLH proteins, fly neurogenesis, and vertebrate myogenesis. Cell 75: 827-830.

Jarman, A.P., Sun, Y., Jan, L.Y., and Jan, Y.N. 1995. Role of the proneural gene, atonal, in formation of Drosophila chordotonal organs and photoreceptors. Development 121: 2019-2030.

Jeon, C.J., Strettoi, E., and Masland, R.H. 1998. The major cell populations of the mouse retina. J. Neurosci. 18: 8936-8946.

Kanekar, S., Perron, M., Dorsky, R., Harris, W.A., Jan, L.Y., Jan, Y.N., and Vetter, M.L. 1997. Xath5 participates in a network of bHLH genes in the developing Xenopus retina. Neuron 19: 981-994.

Morrow, E.M., Furukawa, T., Lee, J.E., and Cepko, C.L. 1999. NeuroD regulates multiple functions in the developing neural retina in rodent. Development 126: 23-36.

Nixon, R.A., Lewis, S.E., Dahl, D., Marotta, C.A., and Drager, U.C. 1989. Early posttranslational modifications of the three neurofilament subunits in mouse retinal ganglion cells: Neuronal sites and time course in relation to subunit polymerization and axonal transport. Brain Res. Mol. Brain Res. 5: 93-108.

Perry, V.H. 1981. Evidence for an amacrine cell system in the ganglion cell layer of the rat retina. Neuroscience 6: 931-944.

Riddle, R.D., Johnson, R.L., Laufer, E., and Tabin, C. 1993. Sonic hedgehog mediates the polarizing activity of the ZPA. Cell 75: 1401-1416.

Tomita, K., Nakanishi, S., Guillemot, F., and Kageyama, R. 1996. Mash1 promotes neuronal differentiation in the retina. Genes Cells 1: 765774.

Wang, S.W., Gan, L., Martin, S.E., and Klein, W.H. 2000. Abnormal polarization and axon outgrowth in retinal ganglion cells lacking the POU-domain transcription factor Brn-3b. Mol. Cell. Neurosci. 16: $141-156$.

Wassle, H. and Boycott, B.B. 1991. Functional architecture of the mammalian retina. Physiol. Rev. 71: 447-480.

White, N.M. and Jarman, A.P. 2000. Drosophila atonal controls photoreceptor R8-specific properties and modulates both receptor tyrosine kinase and Hedgehog signalling. Development 127: 1681-1689.

Young, R.W. 1985a. Cell differentiation in the retina of the mouse. Anat. Rec. 212: 199-205.

- 1985b. Cell proliferation during postnatal development of the retina in the mouse. Brain Res. 353: 229-239. 


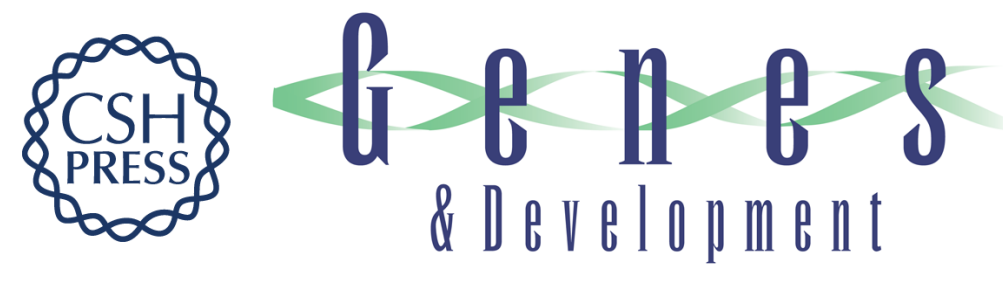

\section{Requirement for math5 in the development of retinal ganglion cells}

Steven W. Wang, Byong Su Kim, Kan Ding, et al.

Genes Dev. 2001, 15:

Access the most recent version at doi:10.1101/gad.855301

References This article cites 29 articles, 13 of which can be accessed free at: http://genesdev.cshlp.org/content/15/1/24.full.html\#ref-list-1

License

Email Alerting Receive free email alerts when new articles cite this article - sign up in the box at the top Service right corner of the article or click here.

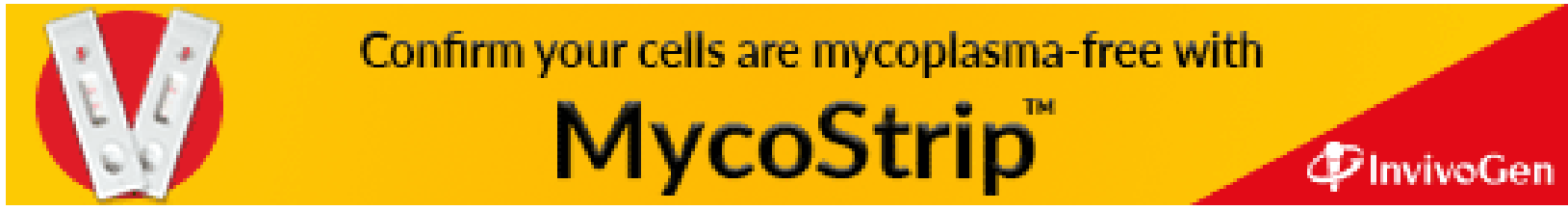

